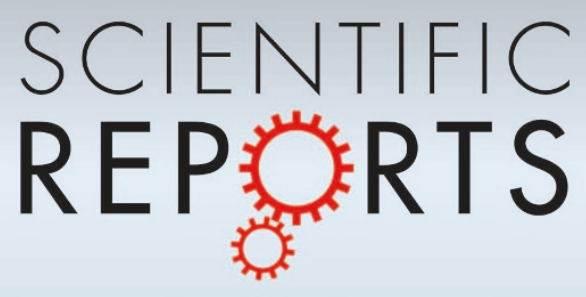

OPEN

SUBJECT AREAS:

POLARITONS

SOLITONS

SUB-WAVELENGTH OPTICS

QUANTUM OPTICS

Received

9 September 2013

Accepted

24 October 2013

Published

12 November 2013

Correspondence and requests for materials should be addressed to

H.H.Y. (haohaiyu@ sdu.edu.cn) or H.J.Z. (huaijinzhang@sdu. edu.cn)

\section{Optical orbital angular momentum conservation during the transfer process from plasmonic vortex lens to light}

\author{
Haohai Yu', Huaijin Zhang', Yicheng Wang', Shuo Han', Haifang Yang' ${ }^{2}$ Xiangang Xu', \\ Zhengping Wang', V. Petrov ${ }^{3} \&$ Jiyang Wang'
}

\begin{abstract}
'State Key Laboratory of Crystal Materials and Institute of Crystal Materials, Shandong University, Jinan 250100, China, ${ }^{2}$ Laboratory of Microfabrication, Institute of Physics, Chinese Academy of Sciences, Beijing, 100190, China, ${ }^{3}$ Max-Born-Institute for Nonlinear Optics and Ultrafast Spectroscopy, 2A Max-Born-Strasse, 12489 Berlin, Germany.
\end{abstract}

We demonstrate the optical orbital angular momentum conservation during the transfer process from subwavelength plasmonic vortex lens (PVLs) to light and the generating process of surface plasmon polaritons (SPPs). Illuminating plasmonic vortex lenses with beams carrying optical orbital angular momentum, the SP vortices with orbital angular momentum were generated and inherit the optical angular momentum of light beams and PVLs. The angular momentum of twisting SP electromagnetic field is tunable by the twisted metal/dielectric interfaces of PVLs and angular momentum of illuminating singular light. This work may open the door for several possible applications of SP vortices in subwavelength region.

S urface plasmon polaritons (SPPs) are generated through strong interaction between electromagnetic field and free electron oscillations on a conductor surface. The free electrons respond and oscillate collectively with the electromagnetic field, and the resonant interaction between the oscillation of surface charges and electromagnetic field results in the unique light scattering and transmitting properties and generate the SP electromagnetic field ${ }^{1}$. The SPPs in general are highly trapped near the surface of the conductor in a small volume in the subwavelength range. Since their recognition in the field of surface science in the $1950 \mathrm{~s}^{2}$, SPP have been of wide interest in fields ranging from physics, chemistry, material science to biology. When light interacts with the metal/dielectric interface of a nanohole or nanoslit, the interference electromagnetic field can be described in terms of Bessel function $s^{3-8}$. In particular, the SP electromagnetic field created by a subwavelength plasmonic vortex lens (PVL) exhibits a spiral phase profile of $\exp (i l \varphi)^{5-8}$, where $l$ is the order of the Bessel functions and $\varphi$ is the azimuthal angle. In the center of the SP electromagnetic field, the phase becomes indeterminate or indefinite as physical quantity and both the real and imaginary parts of this field amplitude vanish which generates a singularity and vortex. The optical angular momentum per photon of SP vortices in the propagation direction $\mathrm{z}$, calculated from the angular momentum operator $\hat{L}_{z}=-i \hbar \frac{\partial}{\partial \varphi}$ is $l \hbar$, therefore, the order $l$ can also be called topological charge and the geometrically twisted pattern of metal/dielectric interfaces would torque the wavefront of the light and bear topological charges. The study of optical angular momentum has stimulated many applications such as particle manipulation, quantum optics, quantum information processing, high-resolution microscopy and lithography ${ }^{9-18}$.

Similar to electrons, photons also possess spin and orbital angular momentum both of them being quantized and capable to transfer torque ${ }^{9,19}$. The spin angular momentum relates to light polarization and for circular polarization, each photon carries angular momentum of $\pm \hbar$ depending on the chirality of the polarization. The orbital angular momentum is determined by the spiral of the optical phase, also called optical vortex, which can carry quantized angular momentum of $\pm j \hbar$ per photon, here, $j$ is an integer. The spin angular momentum of a light beam is intrinsic and the orbital angular momentum is either extrinsic or intrinsic depending on whether the interaction with matter is about an axis where there is or not net transverse momentum ${ }^{20}$. The total angular momentum is conserved during transformations in linear and nonlinear optics ${ }^{17,21-23}$.

Previous investigations showed that SP behavior on a metal surface is similar to light waves in two-dimensional (2D) spaces $^{24-26}$. Thus, it is interesting to investigate whether angular momentum is conserved when the SP electromagnetic field is generated by interaction of a light beam with angular momentum and a conductor with geometrically twisted metal/dielectric interfaces. If angular momentum is conserved, the generated SP angular 


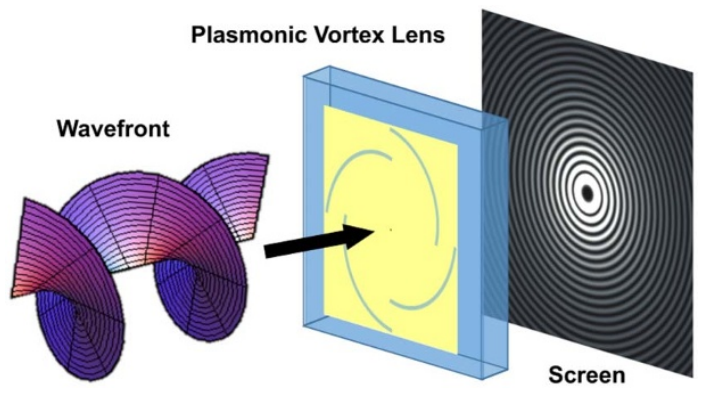

Figure $1 \mid$ Experimental configuration of generated SP vortices. Light beams with the helical wavefront were focused on the surfaces of PVLs. The generated patterns were recorded with a CCD which was placed in a plane immediately behind the plane of the vortex lens as close as possible $(\mathrm{z} \approx 0)$.

momentum per photon could be tuned by the light beam. This would open new possible application in quantum optics and help understand the nature of the SP's angular momentum. However, if the angular momentum is not conserved, residual angular momentum may be acquired by the electrons involved in the interaction which would pave the way for novel optoelectronic devices. However, up to now, the interaction between optical orbital angular momentum and twisted plasmonic nanostructure has not been investigated. Here, we demonstrate optical angular momentum conservation during the process of SP vortices generation by interaction of light beams with orbital angular momentum and geometrically twisted metal/dielectric interfaces. The results prove that the orbital angular momentum per photon of SP vortices inherits the angular momentum of light beams and twisted metal/dielectric interfaces.

\section{Results}

The laser patterns with Laguerre-Gaussian $\left(\mathrm{LG}_{0, l}\right)$ modes with $|l|=0$, 1 , and 2 and unknown chirality were obtained by tuning the pump power of the laser diode ${ }^{27}$. The laser wavelength of $1.08 \mu \mathrm{m}$ corresponded to a SP vortex wavelength of $1.06 \mu \mathrm{m}$ as shown above. Focusing the laser beam on the surfaces of the PVLs as shown in Fig. 1, the SP vortices can be acquired. The patterns of SP vortices obtained with $L G_{00}$ mode and right-handed rotated PVLs with the geometric topological charge $m$ of $-4,-7$ and -10 are shown in Fig. 2 (a), (b) and (c). The intensity distribution of cross sections (red line) through the centers of obtained SP patterns are presented in Fig. 2 (d), (e) and (f). The theoretical intensity distribution equals to $\left|E_{z, n}\right|^{2}$ defined in eq. 2 shown in the Discussion part and is also shown in (d), (e) and (f) (black line) of Fig. 2 with order and topological charge of $n=-4,-7$ and -10 . Since the peaks near the centers are much stronger than the outside ones ${ }^{26}$ and the relative position of peaks is determined by the orders of Bessel and Laguerre-Gaussian functions, we can find that in these figures, the relative position of experimental and theoretical peaks near the centers agrees well in principle, and by which we can determine the order of the Bessel functions and the orbital angular momentum per photon of SP vortices. The discrepancies may be generated by the imperfection of the PVLs, the dispersion of the focal lens and the sensitivity of the CCD.

Increasing the pump power of the laser diode, light beams with $\mathrm{LG}_{0, l}(|l|=1$ and 2) modes were achieved due to the increased nonlinearity including thermal focusing and Kerr effect in the gain medium which decreased the oscillating mode size in the cavity ${ }^{26}$. We found that compared to those shown in Fig. 2, the topological charges $n$ of obtained SPPs increased by 1 or 2 depending on the topological charge $|l|$ of incident light beams. As representative results, the patterns with $|l|=1$ and 2 , and $m=-4$ are shown in (a) and (b) of Fig. 3. The achieved experimental patterns and theoretical analysis of SP vortices with $|l|=1$ and 2 , and $n=-7$ can be found in Supplementary Information. The intensity distribution of cross sections (red line) through the center is presented in the in (c) and (d) of this figure with topological charges of -5 and -6 . From this figure, the total angular momentum per photon of SP vortices is $n=-|l|+m$. The results above also indicate that the chirality of the incident $\mathrm{LG}_{0, l}$ modes is the same as the structure of the PVLs

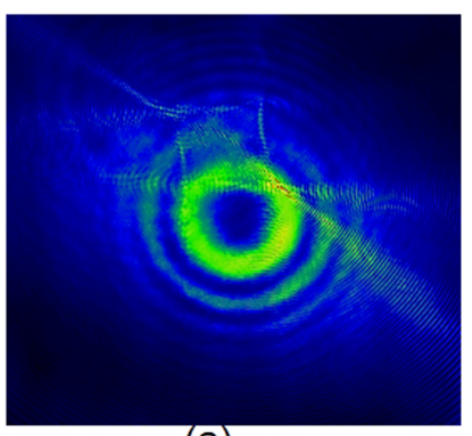

(a)

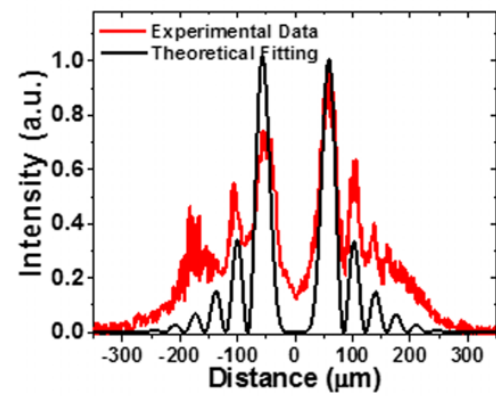

(d)

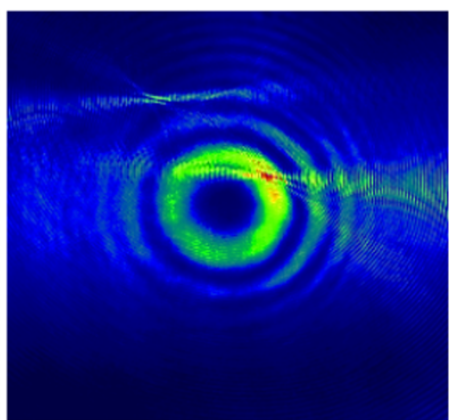

(b)

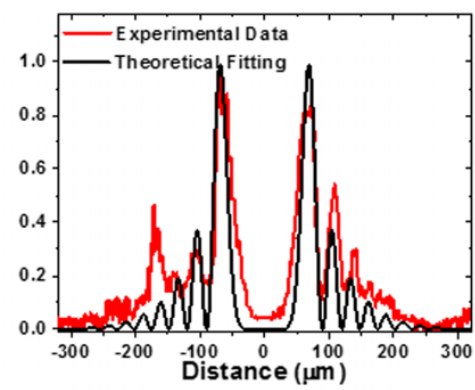

(e)

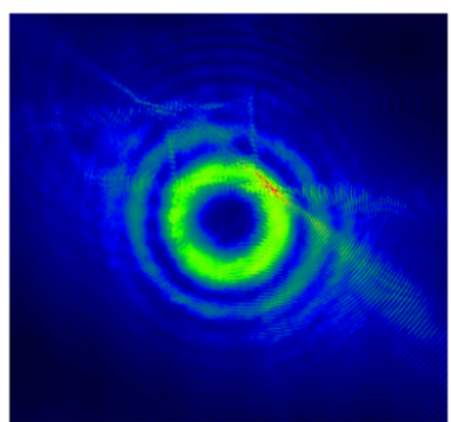

(c)

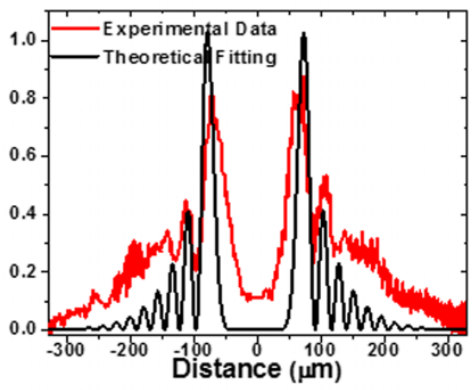

(f)

Figure 2 Experimental patterns and theoretical analysis of the SP vortices. (a)-(c) Experimental patterns of SP vortices using a linearly polarized LG 00 mode and PVL with $\mathrm{m}=-4,-7$ and -10 , respectively. (d)-(f) Experimental data (red lines) of the intensity distribution through the centers of obtained SP vortex patterns corresponding the patterns (a)-(c), respectively, and the theoretical fitting (black lines) of experimental data (red lines) with respective $\left|E_{z, n}\right|^{2}$ functions shown in eq.(2) with $n=-4,-7$ and -10 and the adjusted size of the pattern. 


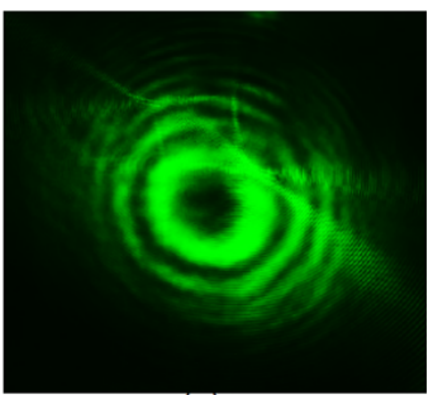

(a)

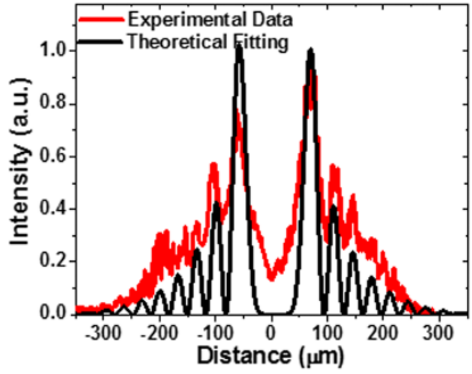

(c)

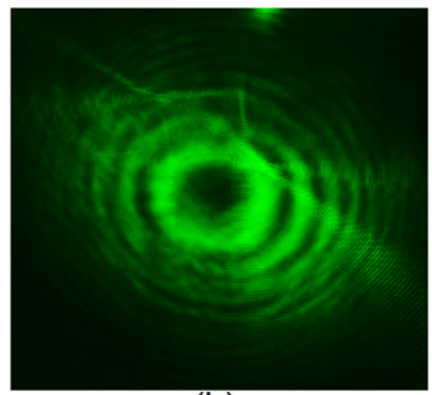

(b)

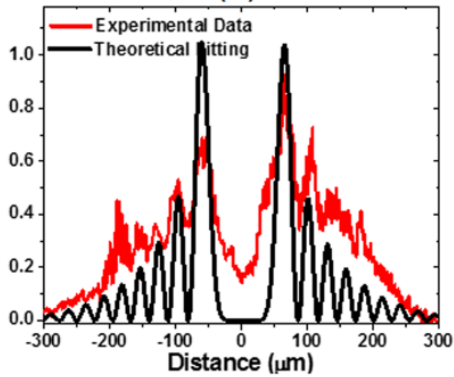

(d)

Figure 3 Experimental patterns and theoretical analysis of the SP vortices using a PVL with $\mathrm{m}=-4$. (a) and (b) Experimental patterns using linearly polarized $\mathrm{LG}_{0,-1}$ and $\mathrm{LG}_{0,-2}$ modes and a PVL with $\mathrm{m}=-4$. (c) and (d) Experimental data (red lines) of the intensity distribution through the centers of obtained SP vortex patterns corresponding the patterns (a) and (b), respectively, and the theoretical fitting (black lines) of experimental data (red lines) with respective $\left|E_{z, n}\right|^{2}$ functions shown in eq. (2) with $n=-5$ and -6 and the adjusted size of the pattern.

which is right-hand rotation and the signs of the topological charge of $\mathrm{LG}_{0, l}$ modes should be minus $(l=-|l|=-1$ or -2$)$. Therefore, the total angular momentum per photon of the SP vortices can be described with $n=l+m$ and the total angular momentum is conserved.

In order to investigate the angular momentum conservation deeply, the mirror symmetry operation for the PVLs was made by inverting the surface of PVLs. The structure of PVLs became left-handed rotation which is opposite to the incident light beams with $\mathrm{LG}_{0, l}$ modes. In this condition, the sign of the PVLs' geometric topological charge $m$ is positive, and the total topological charge is $n=l+m$. As representative results, the patterns with $l=-1$ and $m=10$ are shown in Fig. 4 (a). The intensity distribution of cross sections (red line) through the center are presented in Fig. 4 (b) of this figure with topological charges of 9 . It should be noted that the results show the PVLs can also be used as an optoelectronic device for determination of the topological charge of incident light beams and the total angular momentum is conserved during the process of SPP generation.

Considering the previous results with left- and right-hand polarized light beams with respective spin angular momentum of +1 and -1 and PVLs ${ }^{7}$, the results from this work indicate that similar to light waves in $2 \mathrm{D}$ spaces, the total angular momentum is conserved in all the entire process of SPP generation and the SP vortices should inherit the angular momentum of incident light beams and subwavelength PVLs. SP vortices have been identified to be capable to highly improve the resolution in fluorescence microscopy ${ }^{28}$. This work offers a possible choice for the high-resolution fluorescence microscopy. It can also be expected that this work would unveil the potential of SPPs in possible applications with tunable optical angular momentum per photon.

In conclusion, the optical angular momentum conservation rule is identified for optical vortex beams and PVLs with geometric topological charges. The generated angular momentum per photon of SP vortices is determined and can be tuned by the topological charge of incident vortex beams besides the geometric topological charges of PVLs. It can be expected that this work will unveil the potential of SPPs in the subwavelength region in some aspects.

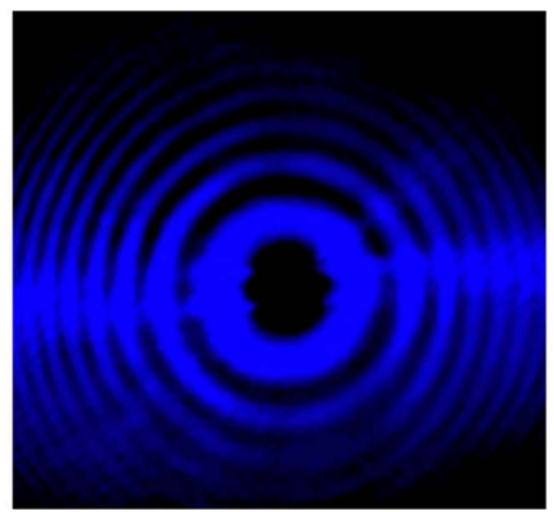

(a)

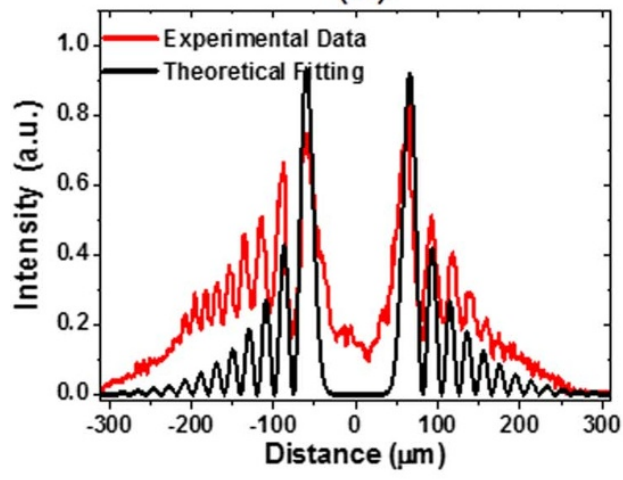

(b)

Figure $4 \mid$ Experimental patterns and theoretical analysis of the SP vortices using a PVL with $\mathbf{m}=10$. (a) Experimental patterns using linearly polarized $\mathrm{LG}_{0,-1}$ mode and a PVL with $\mathrm{m}=10$. (b) Experimental data (red lines) of the intensity distribution through the centers of obtained SP vortex patterns corresponding the patterns (a), and the theoretical fitting (black lines) of experimental data (red lines) with respective $\left|E_{z, n}\right|^{2}$ functions shown in eq. (2) with $n=9$ and the adjusted size of the pattern. 


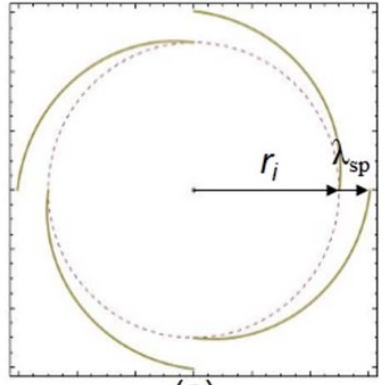

(a)

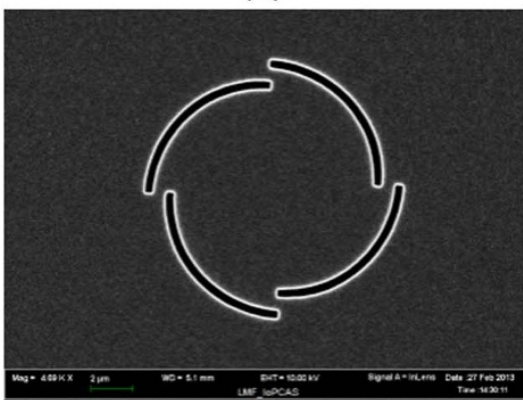

(d)

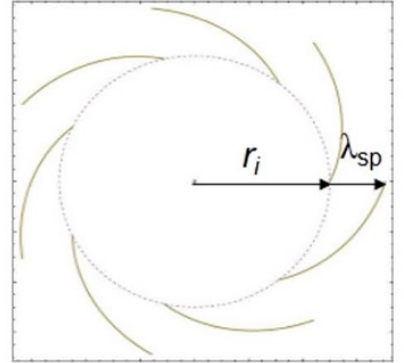

(b)

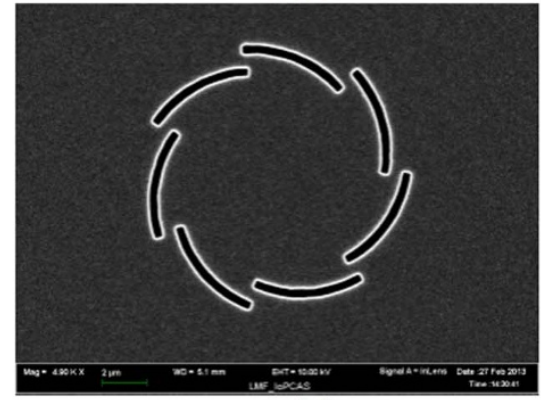

(e)

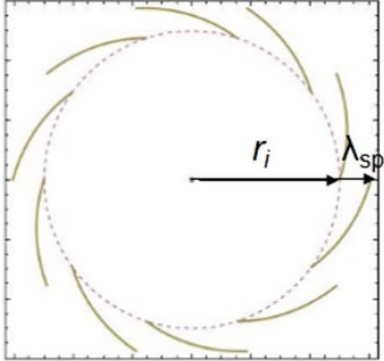

(c)

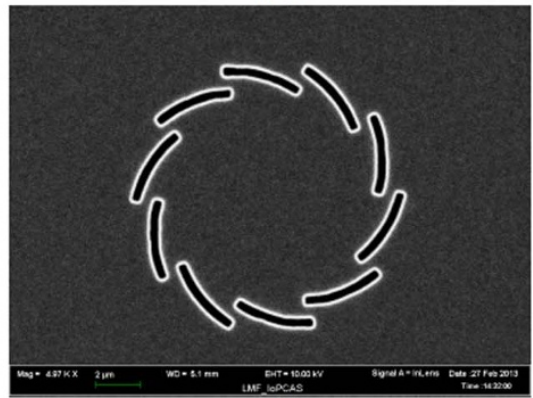

(f)

Figure $5 \mid$ Designed plasmonic vortex lenses. (a), (b) and (c) The theoretical curves of the gold/quartz interface with $\mathrm{m}=4,7$ and 10 , respectively, calculated with eq.3. (d), (e) and (f) The designed PVLs with $\mathrm{m}=4,7$ and 10, respectively.

\section{Discussion}

Only 20 years ago, the Laguerre-Gaussian $\left(\mathrm{LG}_{\mathrm{p}, l}\right)$ laser modes which are eigen modes of a laser cavity described in cylindrical coordinates were recognized to have orbital angular momentum per photon of $l \hbar$ originating from their helical wavefront structure with phase dependence $\exp (i l \varphi)^{9}$. Recently, we reported the direct generation of $\mathrm{LG}_{0, l}$ modes using a pump source with doughnut shaped intensity distribution to enforce the cylindrical symmetry of the generating sys$t_{e m}^{27}$. In the scheme, the orbital angular momentum per photons can be adjusted by the nonlinearity of the medium, e.g. thermal focal or Kerr effects. In the present study of SP vortex generation, the directly generated $\mathrm{LG}_{0 l}$ modes were used as incident light beam as shown in Fig. 1. A polarizer was inserted between the laser and the PVL to keep the linear polarization and zero spin angular momentum per photon of light ${ }^{9}$. The wavelength of the incident laser was centered at $1.08 \mu \mathrm{m}$.

The incident electric field $E^{\text {in }}$ is the $\mathrm{LG}_{p, l}$ laser mode with linear polarization along $\mathrm{X}$ direction $\hat{x}$ :

$$
\begin{aligned}
& E^{i n}(r, \varphi, z) \propto \frac{p}{w} \exp \left[\frac{-i k r^{2} z}{2\left(z_{R}^{2}+z^{2}\right)}-\frac{r^{2}}{w^{2}}\right] \\
& \exp \left[-i(2 p+|l|+1) \arctan \left(z / z_{R}\right)\right] \\
& \times \exp [-i l \phi](-1)^{p}\left(\frac{\sqrt{2} r}{w}\right)^{|l|} L_{p}^{|l|}\left(\frac{2 r^{2}}{w^{2}}\right) \hat{x} .
\end{aligned}
$$

Here, $p$ and $l$ are the radial and azimuthal indexes of the mode respectively, $k$ is the wavenumber of light in free space, $w$ is the beam waist, $z_{R}$ is the Rayleigh rang of the mode, and $L_{p}^{|l|}(x)$ is the Laguerre polynomials. By theoretically analysis shown in Supplementary Information, the corresponding $\mathrm{z}$-directional field electric field of the SP vortices is mathematically represented by the $n$-th order Bessel function $J_{n}(x)$ and Laguerre-Gaussian function with a spiral phase profile given with a scalar equation by:

$E_{z, n}(r, \varphi, z) \propto J_{|n|}\left(k_{s p} r\right) \exp \left[i n\left(\varphi+\frac{\pi}{2}\right)\right] L_{p}^{|l|}\left(\frac{2 r^{2}}{w^{2}}\right) \exp \left(-\frac{r^{2}}{w^{2}}\right)$.
With the angular momentum operator $\hat{L}_{z}=-i \hbar \frac{\partial}{\partial \varphi}$ and eqs. (3) and (4), it can be found that the $L G_{p, l}$ laser mode possesses the orbital angular momentum of $l_{\hbar}$ per photon, and the generated SP vortices have orbital angular momentum of $n \hbar=(l+m) \hbar$ per photon which represents the twisted wavefront. Therefore, PVL can be considered possessing geometric topological charges of $m \hbar$ similar to the quasi-angular-momentum of quadratic nonlinear crystals having a geometrically twisted susceptibility pattern ${ }^{29,30}$. Because the Laguerre-Gaussian mode can keep its form unchanged during propagation and Bessel beam is diffraction-free and self-healing ${ }^{9,31,32}$, the generated SP vortex pattern should have some special propagating properties and the optical angular momentum $n \hbar$ per photon of SP vortices can be achieved by fitting observed patterns with the Bessel and Laguerre-Gaussian functions shown in eq. (2). The intensity of observed patterns is $I(r, \varphi, z) \propto\left|E_{z, n}(r, \varphi, z)\right|^{2}$. Analogous to left- and right-handed circularly polarized light which carries respective spin angular momentum $\pm \hbar$ per photon ${ }^{14}$, geometric topological charges of the left-handed rotated PVLs are defined to be $m$, and those of the right-handed are $-m$.

\section{Methods}

Plasmonic vortex lenses (PVLs) design. The PVLs were designed to satisfy the following equation':

$$
r_{m}(\varphi)=r_{i}+\frac{\lambda_{s p} \bmod (m \varphi, 2 \pi)}{2 \pi} \hat{r}
$$

for $0 \leq \varphi<2 \pi$. Here, $\bmod (m \varphi, 2 \pi)$ represents the remainder of the division of $m \varphi$ by $2 \pi, r_{i}$ is the inner radius defined as the distance from the center to the nearest point of the rim, the radius $r_{m}(\varphi)$ describes the rim of and interface between the gold film and quartz glass substrate with slits of $260 \mathrm{~nm}, \lambda_{s p}=1.06 \mu \mathrm{m}$ is the $\mathrm{SP}$ wavelength, and $\hat{r}$ is the polar unit vector. Since the transmission of the quartz glass substitute is high (about 97\%) for the $1 \mu \mathrm{m}$ light, the recoupling of SPPs and the SP vortex can be negligible. For $m \varphi \leq 2 \pi$ the equation can be shown as:

$$
r_{m}(\varphi)=r_{i}+\frac{m \varphi \lambda_{s p}}{2 \pi}
$$

and the other divisions are the repeat operation on incident light of this division.

For air, the relative dielectric constant $\varepsilon_{\text {air }}=1$, and for substrate, the relative dielectric constant $\varepsilon_{q u a}=3.7$. The relative dielectric of $\mathrm{Au}$ is calculated with a Drude model ${ }^{2}$ : 


$$
\varepsilon_{A u}(\omega)=\varepsilon_{\infty}-\frac{\omega_{D}^{2}}{\omega^{2}+i \gamma_{D} \omega}
$$

Here, $\omega$ is the frequency of the incident laser frequency, and $\varepsilon_{\infty}, \omega_{D}$ and $\gamma_{D}$ are the parameter set to fit empirical data for the real and imaginary part of the gold dielectric constant. For the wavelength at about $1 \mu \mathrm{m}$, the parameters are chosen as $\varepsilon_{\infty}=11.4577$, $\omega_{\mathrm{D}}=9.4027 \mathrm{eV}$ and $\gamma_{D}=0.08314 \mathrm{eV}$. The calculated gold dielectric constant at the wavelength $\lambda_{\text {in }}$ of $1.08 \mu \mathrm{m}$ is $\varepsilon_{A u}=-55.2+i 4.8$. Using the solved Maxwell's equations, the frequency-dependent SP vortices wavelength is ${ }^{3}$ :

$$
\lambda_{S P}=\lambda_{\text {in }} \sqrt{\frac{\varepsilon_{A u}+\varepsilon_{m}}{\varepsilon_{A u} \varepsilon_{m}}} .
$$

The wavelength for a gold-air interface is calculated $\lambda_{\mathrm{SP}}=0.98 \lambda_{\text {in }}=1.06 \mu \mathrm{m}$, at which condition, the momentum mismatching $\Delta k=0$.

The detailed calculation for the design of PVLs and the designed PVLs with $m=4$, 7 and 10 are available in Fig. 5. Analogous to the right- and left-handed rotating polarized light, the PVL slit patterns correspond to right-handed rotation looking from the bottom. The generated pattern of the SP vortices was directly recorded by a charge-coupled device (CCD).

1. Barnes, W. L., Dereux, A. \& Ebbesen, T. W. Surface plasmon subwavelength optics. Nature 424, 824-830 (2003).

2. Ritchie, R. H. Plasma Losses by Fast Electrons in Thin Films. Phys. Rev. 106, 874-881 (1957).

3. Chang, S. H., Gray, S. K. \& Schatz, G. C. Surface plasmon generation and light transmission by isolated nanoholes and arrays of nanoholes in thin metal films. Opt. Express 13, 3150-3165 (2005).

4. Lalanne, P., Hugonin, J. P. \& Rodier, J. C. Theory of surface plasmon generation at nanoslit apertures. Phys. Rev. Lett. 95, 263902 (2005).

5. Lezec, H. J. et al. Beaming light from a subwavelength aperture. Science 297, 820-822 (2002).

6. Lerman, G. M., Yanai, A. \& Levy, U. Demonstration of nanofocusing by the use of plasmonic lens Illuminated with radially polarized light. Nano Lett. 9, 2139-2143 (2009).

7. Kim, H. et al. Synthesis and dynamic switching of surface plasmon vortices with plasmonic vortex lens. Nano Lett. 10, 529-536 (2010).

8. Shen, Z. et al. Visualizing orbital angular momentum of plasmonic vortices. Opt. Lett. 37, 4627-4269 (2012).

9. Allen, L., Padgett, M. J. \& Babiker, M. The orbital angular momentum of light. Prog. Opt. 39, 291-372 (1999).

10. Grier, D. G. A revolution in optical manipulation. Nature 424, 810-816 (2003).

11. Padgett, M. \& Bowman, R. Tweezers with a twist. Nature Photon. 5, 343-348 (2011).

12. Foo, G., Palacios, D. M. \& Swartzlander, G. A. Jr. Optical vortex coronagraph. Opt. Lett. 30, 3308-3310 (2005).

13. Furhapter, S., Jesacher, A., Bernet, S. \& Ritsch, M. Spiral interferometry. Opt. Lett. 30, 1953-1955 (2005).

14. Molina, T. G., Torres, J. P. \& Torner, L. Twisted photons. Nature Phys. 3, 305-310 (2007).

15. Scott, T. F., Kowalski, B. A., Sullivan, A. C., Bowman, C. N. \& McLeod, R. R. Twocolor single-photon photoinitiation and photoinhibition for subdiffraction photolithography. Science 324, 913-917 (2009).

16. Wang, J. et al. Terabit free-space data transmission employing orbital angular momentum multiplexing. Nature Photon. 6, 488-496 (2012).

17. Zürch, M., Kern, C., Hansinger, P., Dreischuh, A. \& Spielmann, Ch. Strong-field physics with singular light beams. Nature Phys. 8, 743-746 (2012).

18. Toyoda, K. et al. Transfer of light helicity to nanostructures. Phys. Rev. Lett. 110 143603 (2013).

19. Němec, P. et al. Experimental observation of the optical spin transfer torque. Nature Phys. 8, 411-415 (2012).
20. O’Neil, A. T., MacVicar, I., Allen, L. \& Padgett, M. J. Intrinsic and extrinsic nature of the orbital angular momentum of a light beam. Phys. Rev. Lett. 88, 053601 (2002).

21. Marrucci, L., Manzo, C. \& Paparo, D. Optical spin-to-orbital angular momentum conversion in inhomogeneous anisotropic media. Phys. Rev. Lett. 96, 163905 (2006).

22. Dholakia, K., Simpson, N. B., Padgett, M. J. \& Allen, L. Second-harmonic generation and the orbital angular momentum of light. Phys. Rev. A 54, 3742-3745 (1996).

23. Mair, A., Vaziri, A., Weihs, G. \& Zeilinger, A. Entanglement of the orbital angular momentum states of photons. Nature 412, 313-316 (2001).

24. Tan, P. S. et al. Phase singularity of surface plasmon polaritons generated by optical vortices. Opt. Lett. 36, 3287-3289 (2011).

25. Raether, H. Surface plasmons on smooth and rough surfaces and on gratings. Springer Tracts Mod. Phys. 111, 1-133 (1988).

26. Gorodetski, Y., Drezet, A., Genet, C. \& Ebbesen, T. W. Generating far-field orbital angular momenta from near-field optical chirality. Phys. Rev. Lett. 110, 203906 (2013).

27. Zhao, Y. G. et al. Direct generation of optical vortex pulses. Appl. Phys. Lett. 101, 031113 (2012).

28. Tan, P. S., Yuan, X. C., Yuan, G. H. \& Wang, Q. High-resolution wide-field standing-wave surface plasmon resonance fluorescence microscopy with optical vortices. Appl. Phys. Lett. 97, 241109 (2010).

29. Bloch, N. V. et al. Twisting light by nonlinear photonic crystals. Phys. Rev. Lett. 108, 233902 (2012)

30. Nikitin, A. Yu., Zueco, D., García-Vidal, F. J. \& Martín-Moreno, L. Electromagnetic wave transmission through a small hole in a perfect electric conductor of finite thickness. Phys. Rev. B 78, 165429 (2008).

31. Durnin, J., Miceli, J. J. Jr. \& Eberly, J. H. Diffraction-free beams. Phys. Rev. Lett. 58, 1499-1501 (1987).

32. Arlt, J. \& Dholakia, K. Generation of high-order Bessel beams by use of an axicon. Opt. Commun. 177, 297-301 (2000).

\section{Acknowledgments}

This work is supported by the National Natural Science Foundation of China (Nos. 51025210, 51102156, 51021062), Grant for State Key Program of China (No. 2010CB630702), the Program of Introducing Talents of Discipline to Universities in China (111 program).

\section{Author contributions}

H.H. Yu and H.J. Zhang contributed equally to this work, conceived the experiments, performed the experiments, collected and analyzed the data, and wrote the paper; H.F. Yang and X.G. Xu prepared the Plasmonic vortex lenses; Y.C. Wang and S. Han performed the experiments; Z.P. Wang, J.Y. Wang and V. Petrov helped with the data analysis, theoretical calculation and paper writing.

\section{Additional information}

Supplementary information accompanies this paper at http://www.nature.com/ scientificreports

Competing financial interests: The authors declare no competing financial interests.

How to cite this article: $\mathrm{Yu}, \mathrm{H}$. H. et al. Optical orbital angular momentum conservation during the transfer process from plasmonic vortex lens to light. Sci. Rep. 3, 3191; DOI:10.1038/srep03191 (2013).

(c) (i) $\Theta$ This work is licensed under a Creative Commons Attribution-

BY NC No Nommercial-NoDerivs 3.0 Unported license. To view a copy of this license, visit http://creativecommons.org/licenses/by-nc-nd/3.0 